\title{
SOBERANÍA ALIMENTARIA Y EL PRINCIPIO CAMPESINO
}

\section{Joost Jongerden ${ }^{1}$ y Guido Ruivenkamp ${ }^{2}$}

\section{Introducción}

El concepto de soberanía alimentaria fue lanzado por las organizaciones de la sociedad civil en la década de los noventa y ha alcanzado un desarrollo importante en la actualidad. Hoy en día, organizaciones internacionales como la FAO se hallan comprometidas con el debate sobre soberanía alimentaria. Esto, por un lado, es una muestra del éxito del concepto y de la aparente necesidad de ir más allá de las complicadas ofertas existentes, como las de la alimentación como derecho humano y de la seguridad alimentaria. Por otro lado, está presente el riesgo de que el concepto de soberanía alimentaria sea despojado de su confrontante contenido, e integrado en los "negocios como habitual". Este artículo discutirá el movimiento de soberanía alimentaria como un principio orde- nador alternativo, capaz de confrontar a la organización económica internacional existente y capaz de ir más allá de las ofertas de alimentación como un derecho humano y de seguridad alimentaria. Después de una breve discusión sobre los antecedentes -la persistencia del hambre y la pobreza y la amplia brecha entre ricos y pobres, parte del actual sistema económico mundial- la soberanía alimentaria es discutida dentro del contexto de una re-campesinación de los desarrollos agrícolas. Se argumenta que el concepto de soberanía alimentaria nos permite ir más allá de la modernización agrícola, y del principio campesino en agricultura. Concluimos: el concepto de soberanía alimentaria nos obliga a pensar sobre alternativas en el desarrollo, no sólo en el mercado internacional, sino también en otros ámbitos, por ejemplo, en la formulación

1 Joost Jongerden es profesor asistente en el Critical Technology Construction Group de la Universidad Wageningen. Contacto: CTC-WUR, Hollandseweg 1, 6706 KN Wageningen, the Netherlands. joost.jongerden@wur.nl

2 Guido Ruivenkamp, profesor en el Athena Institute, Vrije Universiteit Amsterdam y profesor asociado en el Critical Technology Construction Group de la Universidad Wageningen. Contacto: CTC-WUR, Hollandseweg 1,6706 KN Wageningen, the Netherlands.guido.ruivenkamp@wur.nl 
de agendas de investigación y desarrollo tecnológico.

\section{Antecedentes}

Hoy en día, cerca de 2.8 billones de personas; es decir, el $45 \%$ de la población mundial vive por debajo de la línea de pobreza, con un ingreso de 2 USD al día, y más de 1.1 billones de personas, valor equivalente a casi el $18 \%$ de la población mundial, se ubica por debajo de la línea de pobreza con un 1 USD al día (Woodward \& Sims, 2006: 1). Mientras, al menos, la mitad de la población mundial vive en extrema pobreza, sin embargo, existe una riqueza sin precedentes entre los ricos del mundo. El 1\% más rico del mundo recibe tanto como el $57 \%$ más pobre. Y los 25 millones de americanos ricos tienen el mismo ingreso económico que casi 2 billones de la población más pobre del mundo (Milanovic, 2002: 51-92). No solamente hay una desigualdad en la distribución del ingreso económico, sino que la desigualdad global también se incrementó. Un análisis de tendencias a largo plazo muestra la distancia entre los países más ricos y los más pobres del mundo, la cual estaba cerca de 3 a 1 en $1820 ; 11$ a 1 en 1913; 35 a 1 en 1950; 44 a 1 en 1973; 72 a 1 en 1992 (Human Development Report, United Nations Development Program, 1999). Esto está relacionado con la fuga de capital y la desigualdad en el crecimiento económico. Con relación a la fuga de capital, los datos proporcionados por el Banco Mundial revelan que los países en desarrollo gastan 13 USD en prepago de deuda por cada dólar que reciben en donaciones (Global Development Finance, World Bank, 1999)33. El beneficio promedio del crecimiento global para el $1 \%$ más rico de la población podría ser hasta 120 veces más de lo que es para el 10\% más pobre. Estos datos ilustran la desigualdad producida por el crecimiento económico. Si la tendencia de años anteriores ha continuado, la proporción del ingreso económico entre el $1 \%$ más rico y el $10 \%$ más pobre debió llegar en el 2006 al orden de 170 veces más (Woodward \& Sims, 2006: 12). El crecimiento tiene una predisposición pro-rico, contribuye más al rico que al pobre, y agranda la brecha entre ricos y pobres. Los acuerdos de libre comercio no con- 
tribuyen a mejorar esta situación, más bien la empeoran. Justamente, el movimiento de soberanía alimentaria emerge como una respuesta a los intentos de actores estatales por ampliar los convenios de libre comercio en agricultura, amenazando el estilo de vida de millones de campesinos y de otros quienes se ganan la vida en el campo.

\section{Soberanía alimentaria}

La soberanía alimentaria, se ha convertido en un concepto central en los movimientos campesinos y en las organizaciones de la sociedad civil que están preocupadas por la reforma agraria y el desarrollo (ICARRD, 2006: 5). El movimiento campesino internacional Vía Campesina ${ }^{4}$ desarrolló el concepto y lo introdujo dentro del debate público durante la Cumbre Alimentaria Mundial en 1996. Durante la pasada década, el concepto se ha vuelto un tema mayor en el debate agrícola internacional. El tema de soberanía alimentaria es el propuesto por la Red de Soberanía Alimentaria del Pueblo del 2002 "People's Food Sovereignty Network" (Windfuhr \& Jonsen, 2005).

Soberanía Alimentaria es el derecho de los pueblos a definir su propia alimentación y agricultura para proteger y regular la producción agrícola doméstica y comercializarla con la finalidad de alcanzar objetivos de desarrollo sostenible y así determinar el punto en el cual quieren ser autosuficientes para restringir el arrojo de productos en sus mercados y proveer a las comunidades basadas en pesqueras locales la prioridad en el manejo del uso de y los derechos de los recursos acuáticos. La soberanía alimentaria no niega el comercio, sino que más bien promueve la formulación de políticas y prácticas de comercio que sirven a los derechos de los pueblos para alimentar y asegurar una producción sostenible saludable y ecológica.

Las ideas claves son el derecho a definir las políticas y agriculturas

4 Vía Campesina fue establecida en 1993 en Bélgica. La organización se define a sí misma de la siguiente manera:"Somos el movimiento internacional de campesinos, productores pequeños y medianos, sin tierras, mujeres rurales, pueblos indígenas, juventud rural, y trabajadores agrícolas. Defendemos los valores y los intereses básicos de nuestros miembros. Somos un movimiento autónomo, pluralista y multicultural, independiente de cualquier afiliación política, económica, o de otro tipo. Nuestros miembros son de 56 países de Asia, África, Europa, y las Américas." http://www.viacampesina.org/main_en/index.php?option= com content\&task=blogcategory\&id=27\&ltemid=44, fecha de acceso 17-09-2008 
propias para proteger y regular mercados, y el desarrollo de prácticas que hagan efectivo "el derecho a la alimentación". El concepto de soberanía alimentaria surgió como una respuesta a negociaciones dentro de la Organización Mundial de Comercio (OMC) para la liberación de los mercados, y del control de la agricultura (para una discusión ver Lee, 2007). Los acuerdos de libre comercio realizados por algunos Estados, no les permitirán más a estos Estados el proteger sus mercados internos, sino que les obligarán a organizar producciones agrícolas en una ideología de libre comercio.

Las políticas neoliberales aspiran a un sistema de producción global organizado en cadenas, y le otorgan la agencia a las corporaciones globales y a las empresas agrícolas comerciales. El concepto de soberanía alimentaria fue lanzado para proporcionar una alternativa a las políticas neoliberales, a través de un paquete de políticas destinadas al desarrollo rural con el fin de contribuir a la reducción del hambre y la pobreza. La producción y disponibilidad de alimentos debían ser aprovisionadas por los sistemas alimenticios locales y los campesinos debían ser su soporte. Las recomendaciones políticas enfatizaron, principalmente, en el derecho a definir políticas nacionales (en oposición a la WTO y a los tratados de libre mercado) y a efectuar reformas agrarias (en oposición a la desigualdad de la distribución de tierra, tales como el patrón latifundio/minifundio en muchas partes de América Latina y a severas limitaciones a los recursos productivos de los productores agrícolas) (ICARRD, 2006: 6). La construcción de sistemas agroalimentarios flexibles a nivel local va emparejada con una política de equidad en (y por) el desarrollo rural y la protección para evitar los acuerdos de libre mercado y el dumping 5 .

El concepto de soberanía alimentaria también debe ser entendido en el contexto más general del derecho a una adecuada alimentación y del concepto de seguridad alimentaria. El derecho a una adecuada alimentación, como uno de los derechos humanos, es reconocido en varios instrumentos internacionales. Se argumenta que el derecho a una adecuada alimentación está indivisiblemente vinculado a la inherente dignidad 
de la gente y es indispensable para la obtención de otros derechos humanos incluidos en el Acta Internacional de los Derechos Humanos (United Nations Economic and Social Council 1999). En el Acuerdo Internacional sobre Derechos Económicos, Sociales y Culturales de 1966, el derecho a una alimentación adecuada se trata dentro del contexto del derecho a una autodeterminación y el derecho a determinar su estatus político y buscar libremente su desarrollo económico, social y cultural. El Tratado declara en el artículo 11.1 que los Estados firmantes reconocen el "derecho de cada uno a un estándar adecuado para desarrollar su propia vida y la de su familia, incluyendo una adecuada alimentación, ropa y techo, y al continuo mejoramiento de las condiciones de vida", mientras el artículo 11.2 se refiere al "derecho fundamental a ser libres de hambre y la malnutrición" (International Covenant on Economic, Social and Cultural Rights 1966).

A pesar de que la comunidad internacional ha reafirmado frecuentemente la importancia del derecho a una adecuada alimentación, la bre- cha entre los derechos establecidos y la situación prevaleciente en el mundo está creciendo, con 840 millones de personas crónicamente hambrientas en 1999 (cuando la ONU reafirmó el derecho a una alimentación adecuada). El encargado especial de la ONU sobre el derecho a la alimentación, Jean Ziegler, emitió una declaración sobre la ocasión del Día Mundial de la Alimentación de este año a ser celebrado el 16 de octubre de 2007, en que el número de gente sufriendo de hambre se había incrementado cada año desde 19966. El problema de hambre, que la comunidad internacional reconoce, no es un problema de disponibilidad, sino de accesibilidad (United Nations Social and Economic Council, 1999). De acuerdo con la FAO, la producción de comida es suficiente para alimentar a 12 billones de personas, el doble de la población mundial actual.

El derecho a una adecuada alimentación impone tres niveles de obligaciones sobre los Estados que son parte. Estas obligaciones son para respetar, proteger y hacer efectiva la ley. "La obligación de respetar el acceso (ya existente) a una adecuada 
alimentación requiere que los Estados parte no tomen ninguna medida que obstruya dicho acceso. La obligación para proteger requiere medidas de los Estados para asegurar que las empresas o individuos no priven a otros individuos del acceso a una adecuada alimentación. La obligación de facilitar significa que el Estado debe comprometerse proactivamente en actividades con la intención de fortalecer los accesos de la gente a una utilización de los recursos y los medios para asegurar su vida, incluyendo seguridad alimentaria (Un Economic and Social Council 1999: art. 15)".

El principal enfoque de la comunidad internacional para implementar el derecho a la alimentación han sido las ideas de "seguridad alimentaria". El concepto de seguridad alimentaria es ciertamente ambiguo, evolucionó en los últimos treinta años y refleja los cambios en el pensamiento político internacional (Clay, 2002; Heidhues et. al., 2004). El término se acuñó a mediados de la década del setenta, cuando la Conferencia Mundial sobre la Alimentación (1974) definió la seguridad alimentaria como la posibili- dad de asegurar la disponibilidad de la comida. En 1983, los análisis de la FAO se enfocaron en el acceso a la comida. En la Cumbre Mundial sobre la Alimentación (1996) se definió lo que significaba la situación de seguridad alimentaria (FAO Policy Brief, 20067): "La seguridad alimentaria existe cuando toda la gente, en todos los momentos, tiene acceso físico y económico a una alimentación suficiente, segura y nutritiva que cumpla con sus necesidades dietéticas diarias y con sus preferencias alimentarias y permita una vida activa y saludable" (World Food Summit, 1996). La definición enfatiza disponibilidad y acceso a una alimentación adecuada en todo momento.

Sin embargo, durante las décadas pasadas, las políticas de ajuste estructural han reducido los servicios públicos y la habilidad de los gobiernos (locales) para lidiar con la inseguridad alimentaria, debilitando a los países en desarrollo para que puedan combatir efectivamente con el hambre y la pobreza. Estas, -así llamadas- políticas de ajuste no solamente implican una pérdida en la soberanía, sino también una pérdida 
en términos de democracia puesto que son las agencias internacionales y no la gente quienes determinan las políticas que afectan sus vidas. De manera creciente, las organizaciones internacionales (tales como FAO y las ONG internacionales) se han convertido en los actores principales que bregan con la repentina crisis. Como consecuencia, la seguridad alimentaria se ha tornado en una estrategia intervencionista, trayendo comida de cualquier otra parte para luchar con situaciones de inseguridad alimentaria (Mousseau, 2005: 20-21).

El concepto de soberanía alimentaria, va más allá del derecho humano a la comida, tal como ha sido determinado por la ley internacional, y más allá de la propuesta de seguridad alimentaria. En los acuerdos internacionales, el Estado es parte, no son los ciudadanos, y la agencia para lograr la realización del derecho humano a una adecuada alimentación está ubicada en el Estado. Sin embargo, los Estados (como resultado de los acuerdos internacionales y de las políticas de ajuste) están perdiendo el espacio político para manejar sus propias políticas que aseguren la ejecución efectiva de las leyes y los derechos internacionales acordados. En este sentido, la oferta de seguridad ali- mentaria es meramente una oferta técnica: es acerca de la disponibilidad y acceso, pero no dice nada acerca de dónde viene la comida o cómo se produce. Cuando la comida es suministrada como una estrategia de alivio al hambre, la oferta de seguridad alimentaria está, posiblemente, contribuyendo a una creciente inseguridad alimentaria porque disminuye la capacidad de producción local (Mousseau, 2005: 22).

\section{Más allá de la moderniza- ción/desarrollo}

Una característica importante del concepto y movimiento de soberanía alimentaria es que desafía al actual sistema de producción alimentaria (un sistema de producción organizado en cadenas) y está moldeado a los acuerdos y tratados internacionales. Una cadena, podríamos decir, se caracteriza por la integración vertical de los actores. Las cadenas están organizadas globalmente a través de un centro de comando, el actor central es quien coordina el flujo de información y de productos. La industria de procesamiento tenía el rol de centro de comando, pero gradualmente esta posición estratégica ha sido tomada por los comerciantes, quienes tienen la función estratégica de distribución hacia los consumidores. 
Estas cadenas producen una desconexión de los sistemas de consumo y producción local y generan en el establecimiento de flujos globales de comida y servicios. Las políticas están diseñadas para facilitar este flujo internacional organizado de comida y servicios. En este modelo de desarrollo los campesinos son considerados un obstáculo, y son quienes deben ser transformados en productores empresariales, quienes deben tener su producción y reproducción en consonancia con la lógica de estas cadenas globales, o deben abandonar la producción agrícola.

En otro artículo Jongerden (2008) analiza la transformación de los sistemas de producción campesina en sistemas de producción empresarial como un proceso de destrucción creativa e ilustra esto discutiendo el libro The Grapes of Wrath de John Steinbeck y The Legend of the Thousand Bulls (Binbo_alar Efsanesi) de Ya ar Kemal. La historia del libro de Kemal, publicada en 1971, está ubicada en Turquía en la década del cincuenta, periodo en que Marshall impulsó la mecanización y se empezó a llevar a la modernidad hacia el campo. La principal preocupación de la historia son los nómadas de Aydinli, una rama de los Yörük. La historia explica cómo los Aydinli, que una vez fueron una poderosa tribu nómada con no menos de 2.000 tiendas negras, se han convertido ahora en un disminuido y marginado grupo. La creación de modernos sistemas de agroempresas los está exprimiendo, y aunque el desarrollo está beneficiando a algunos de ellos, el dolor es el común para la mayoría. La pregunta genérica que se levanta de este ejemplo en particular es ¿Cómo lograr un tipo de desarrollo que beneficie no sólo a algunas personas y perjudique a la mayoría? Prominentes pensadores, tales como Wolfgang Sachs, afirmarán que esta es la pregunta errónea y que (este) desarrollo es una ilusión. Acorde con Sachs, quien rechaza la idea de desarrollo (Desarrollo con ' $D$ ' mayúscula, con su discurso y práctica incluido dentro de las organizaciones internacionales), enfatiza que el constante desarrollo y lo que lo acompaña ha llevado a la desilusión, decepción, ruina y crimen y, por lo tanto, no debemos temerle al fracaso del desarrollo, sino a su éxito (Sachs, 1992: 1-5).

La noción de destrucción creativa simplemente sostiene que la creación de nuevos patrones de desarrollo conlleva la destrucción de arreglos previos. La idea de destrucción creativa se remite al trabajo de Karl Marx y ha sido discutida por muchos pensadores diferentes tales como Walter 
Benjamin y Joseph Schumpeter. Benjamin escribe en su famoso Work of Art in the Age of Reproducibility, del cual una primera versión fue escrita en 1936, que la modernidad es "inconcebible sin su lado destructivo, catártico: la liquidación del valor de tradición ${ }^{8 ”}$ (Benjamin, 2003 [1936]: 254).

Schumpeter escribe en una publicación de 1942 que las economías modernas "revolucionan la estructura económica desde adentro, destruyendo incesantemente la antigua, creando continuamente una nueva. Este proceso de Destrucción Creativa es el hecho esencial acerca del capitalismo" (Schumpeter 1975 [1942]: 83-84).

Este proceso de destrucción creativa es más que una ley anónima de desarrollo capitalista, está también incluida en políticas, entre otras las políticas de libre comercio a las que se opone el movimiento de soberanía alimentaria. En un análisis de neoliberalismo, David Harvey afirma que el dinero liberalismo, actualmente, ha tenido una limitada efectividad como una máquina para el crecimiento económico, pero que ha sido exitoso en desafiar la abundancia de las clases subordinadas hacia las dominantes y de los países pobres a los ricos. Harvey describe esto como un proceso de destrucción creativa que "implica el desmantelamiento de instituciones y de narrativas que promovieron medidas distributivas más igualitarias que en la era precedente" (Harvey, 2007: 22). Esto incluye la destrucción los acuerdos Keynecianos y social demócratas, la habilidad de los Estados para implementar políticas sociales, y la creación de sistemas de libre mercado. Los acuerdos neoliberales preparados por los Estados en un escenario de la Organización Mundial del Comercio

8 Por supuesto,uno puede argumentar que la misma idea de destrucción productiva ya ha sido presentada por Karl Marx y Friedrich Engels en 1848 en el Manifiesto Comunista. "La burguesía no puede existir sin revolucionar constantemente los instrumentos de producción, y por eso las relaciones de producción, y con ellas todas las relaciones de la sociedad. La conservación de los antiguos modos de producción en forma inalterada, eran, por el contrario, la primera condición de existencia para todas las clases industriales iniciales. La constante revolución de la producción, la ininterrumpida perturbación de todas las condiciones sociales, la eterna incertidumbre y agitación distinguieron la época burguesa de todas las anteriores. Todas las fijas, rápidamente congelar las relaciones, con su tren de antiguos y venerables prejuicios y opiniones, son borrados, todo lo que se ha formado se convierte en anticuado antes de que se pueda osificar. Todo lo que es sólido se derrite en el aire, todo lo que es sagrado es profano, y el hombre es finalmente confrontado cara a cara con sobrios sentidos con su condición real de vida y sus relaciones con su género". http://www.marxists.org/archive/marx/works/1848/communist-manifesto/ch01.htm 
(OMC) son considerados acuerdos de destrucción creativa: bien sea intencionados, o en sus efectos, para destruir sistemas de producción campesinos, mientras simultáneamente desarrollan granjas ${ }^{9}$ empresariales y corporativas.

\section{El principio campesino}

El movimiento de soberanía alimentaria trae al centro del debate, el tema de lo que podríamos llamar el "principio campesino" (Van der Ploeg, 2008). Aquéllos, quienes expresan la necesidad por soberanía alimentaria, están involucrados en el movimiento campesino. El principio campesino también enfatiza en la importancia de las redes locales alimentarias, en oposición al concepto de las cadenas alimentarias (globales). Estas redes son de considerable importancia para la producción y disponibilidad de comida en importantes sectores de la población: ellas están compuestas por una multitud de medianas y pequeñas empresas (familiares), y mucho del consumo y producción toma lugar dentro de la red. La hipótesis central es que estas cadenas son capaces de producir y proveer comida debido a la estrecha articulación entre consumo y producción. No estamos sugiriendo que estas cadenas sean autárquicas. De hecho existen relaciones con otros centros de producción y consumo en todas partes pero ésta no es una característica principal de su trabajo $y$ es la diferencia principal con el modelo de cadena. Esto implica que la lógica de la cadena es su incrustación en los procesos locales (producción y consumo). El segundo presupuesto, relacionado con el anterior, es que estas cadenas están principalmente organizadas dentro de una geografía bien definida (aunque fluida). Nuevamente, la red no es un enclave y los vínculos con otras geografías existen. Pero la localidad es el marco principal de referencia para la cadena.

9 La distinción entre campesinos, y financieros empresariales y corporativos viene de Jan Douwe van der Ploeg (2008). En los sistemas de producción campesinos la producción está orientada hacia el mercado y la reproducción de la granja, mientras el trabajo es provisto básicamente por la familia (movilizado en la comunidad a través de relaciones de reciprocidad), y los mayores medios de producción están bajo propio control. La granja empresarial está construida bajo capital financiero e industrial, y la producción es especializada y completamente orientada hacia el mercado. En la granja capitalista corporativa la protrusión está orientada exclusivamente hacia el mercado y la fuerza de trabajo compuesta de trabajadores asalariados 
Hemos definido agricultura campesina como un sistema de producción en el cual el trabajo es básicamente provisto por la familia (o movilizado dentro de la comunidad a través de relaciones de reciprocidad), la tierra y los medios de producción pertenecen a los productores, la producción está orientada hacia el mercado y los recursos son producidos y reproducidos dentro de la unidad de producción (Van der Ploeg, 2008: 20-22; 29). El principio campesino, es un principio de orden, el cual toma como punto de referencia las luchas campesinas (agencia) y sistemas de producción campesina (una relación particular con el trabajo, mercados y naturaleza). Es una lucha por la autoregulación y la autonomía, lo cual puede ser considerado una alternativa a los esquemas regulatorios dominantes (principios de orden). Generalmente, estos esquemas de regulación son promovidos por agroindustrias y el Estado, y contribuyen a la realización de mercados anónimos y al control a distancia de la producción agrícola (Van der Ploeg, 2008: 261-299; Ruivenkamp, 1989). Este control a distancia es parte de las relaciones de dependencia en la cadena, y se efectúa a través de créditos, aportes químicos y semillas, entre otros. En este sentido, el principio campesino "representa una poderosa salida de la creciente y multidimensional crisis agraria global que estamos atestiguando actualmente" (ibíd: 278). Como tal, el principio podría funcionar como un vínculo entre la "construcción de una nueva autonomía" y "la promesa de que las cosas pueden mejorar a través de la dedicación propia y la voluntad de comprometerse en la luchas diarias ${ }^{10 "}$ (Van der Ploeg, 2008: 277).

Considerando los antecedentes neoliberales en contra de los cuales el concepto de soberanía alimentaria y el movimiento emergieron, $y$ tomando en cuenta la amenaza a los campesinos y a sus sistemas de producción, por las políticas de libremercado, no es sorprendente que el concepto de soberanía alimentaria haya surgido primero como un marco de política y un discurso, una respuesta a la inclusión de la agricultura dentro del sistema de mercado mundial. La demanda inicial era el derecho "a producir nuestra propia comida en nuestro propio territorio" (Vía

10 Como ejemplos, Van der Ploeg se refiere al Movimento dos Sem Terra (MST) en Brasil y las ondas iniciales en el movimiento de granjeros orgánicos. 
Campesina, citada en: Lee, 2007), llamando a los gobiernos a rechazar la propuesta de la OMC para la liberación y protección de los mercados.

Sin embargo, en el 2002, Vía Campesina lanzó una propuesta para un campesino sostenible, basado en producción agrícola en la Cumbre Mundial sobre Desarrollo Sostenible en Johannesburgo. Luego de haber observado que el mayor obstáculo para el desarrollo es la forma en cómo las políticas internacionales y nacionales, así como también el agronegocio fuerza a los pequeños productores a adoptar métodos de producción sostenible a través de un modelo de competencia e industrialización, que no toma en cuenta la producción familiar; Vía campesina expone varias propuestas: una de ellas se refirió a la necesidad de desarrollar una infraestructura rural apropiada para una agricultura de bajo aporte externo y la producción para mercados domésticos. Adicionalmente, surgió una preocupación por la investigación y la tecnología. El desarrollo de la investigación y de la tecnología apoyan principalmente a los sistemas de industrialización y alimentación global de la descampesinización de la agricultura, podríamos decir. Sin embargo, se ha argumentado, que la investigación agrícola también podría empe- zar desde el sistema de producción local. Esto podría cubrir varios temas. Así Quaye se refiere a políticas de soberanía alimentaria relacionadas con semillas. Ella argumenta que la política de semillas no debería ser restringida de conservar antiguas variedades, sino de desarrollar nuevas en concordancia con los deseos de los productores y consumidores locales. En otras palabras, es tomar la soberanía alimentaria seriamente, también implica que necesitamos reconsiderar las existentes prioridades de investigación y desarrollo de tecnologías. Ruivenkamp (2008) argumenta que necesitamos revisar nuestro entendimiento y relación con la tecnología. El desarrollo de tecnología es un proceso social y por lo tanto, debe estar incrustado firmemente en el dominio social, esto implica, que el desarrollo tecnológico debe tomar lugar en una constante interacción con las partes interesadas, las cuales, como consecuencia, están desarrollando una relación con la tecnología como co-diseñadores.

\section{Observaciones finales}

Inicialmente, el concepto de soberanía alimentaria parecía priorizar la protección sobre el libre comercio, y como tal al Estado sobre el mercado. Todavía los tratados de 
Libre Comercio son establecidos y supervisados por los Estados, y no existe contraindicación intrínseca entre los mecanismos de libre mercado y las políticas gubernamentales. Por el contrario, la creciente política estatal neoliberal se ha convertido en instrumental para el posterior desarrollo del liderazgo de libre comercio por lo menos para la reciente crisis financiera y la necesidad de nuevas intervenciones del Estado. La característica más interesante del movimiento de soberanía alimentaria, es que éste representa una nueva posibilidad para los políticos, más allá de la Nación-Estado y el libre comercio, más allá de la paradoja de Estado y mercado. En palabras de Hardt y Negri, éste representa el poder de la multitud para crear alternativas. Confrontar la realidad existente con una multitud de iniciativas creando otras realidades socio-técnicas, y personifica la promesa y posibilidad de que otro mundo es posible (2004).

\section{Bibliografía}

BENJAMIN, Walter

2003 Selected Writings, Volume 4, 1938-1940. Cambridge \& London: Prensa de la Universidad de Harvard.
COTULA, Lorenzo y Margret Vidar

2002 The Right to Adequate Food in Emergencies, FAO Estudio Legislativo 77. Roma: FAO

HARDT, Michael y Toni Negri

2004 Multitude, war and democracy in the age of Empire. New York: The Penguin Press

HARVEY, David

2007 Neoliberalism as Creative Destruction, The ANNALS of the American Academy of Political and Social Science 2007; pp. 610-21.

International Conference on Agrarian Reform and Rural Development ICARRD

2006 Agrarian Reform in the Context of Food Sovereignty, the Right to Food and Cultural Diversity: Land, Territory and Dignity.

JONGERDEN, Joost

2008 "First the Peasant. Some Reflections on Modernity, Technology and Reconstruction". In: Guido Ruivenkamp, Shuji Hisano \& Joost Jongerden. Reconstructing Biotechnologies, critical social analyses. Wageningen: Wageningen Academic Publishers.

MILANOVIC, Branko

2002 True World Income Distribution, 1988 and 1993: First Calculation Based on Household Surveys Alone, Economic 


\section{Joost Jongerden y Guido Ruivenkamp}

Journal, Royal Economic Society, vol. 112(476), pp. 5192.

MOUSSEAU, Frederic y Anuradha Mittal

2005 Food Aid or Food Sovereignty, ending world hunger in our time. The Oakland Institute.

RUIVENKAMP, Guido

1989 De Invoering van Biotechnologie in de Agro-Industriele Productieketen. Utrecht: Jan van Arkel.

RUIVENKAMP, Guido

2008 And Joost Jongerden. From prescription to reconstruction: Opportunities for subpolitical choices in biotechnological and genomics research. Presentación en el International seminario "Towards a Lingua Democratica" en la Universidad Humanística, Utrecht, The Netherlands, Octubre 9-11, 2008.

SACHS, Wolfgang (ed)

1992 The Development Dictionary: A Guide to Knowledge as Power. London: Zed.

SCHUMPETER, Joseph

1975 [1942]. Capitalism, Socialism, and Democracy. New York: Harper.

United Nations Economic and Social Council

1999 The right to adequate food (Art.11): 12/05/99. E/C.12/ 1999/5 (Comentarios Generales)
United Nations

1966 International Covenant on Economic, Social and Cultural Rights Adoptado y abierto para firma, ratificación y adhesion por resolución General de la Asamblea 2200A (XXI) de 16 de diciembre de 1966

VAN DER PLOEG, Jan Douwe

2008 The New Peasantries, struggles for autonomy and sustainability in an era of Empoire and Globalization. London: Earthscan.

WINDFUHR, Michael and Jennie Jonsén

2005 Food Sovereignity, towards democracy in localized food systems. ITDG Publishing.

WOODDWARD, David \& Andrew Sims

2006 Growth isn't Working. The unbalanced distribution of benefits and costs from economic growth. London: New Economics Foundation.

\section{Bibliografía de Internet}

Global Development Finance, World Bank, 1999 http://web.worldbank.org/ WBSITE/EXTERNAL/EXTDEC/EXTDECPROSPECTS/EXTGDF/EXTGDF19 99/0,,menuPK:544612 pagePK:6416770 2 piPK:64167676 theSitePK:544595,00 .html) 


\section{Soberanía alimentaria y el principio campesino}

Human Development Report, United Nations Development Program 1999 (http://hdr.undp.org/en/reports) LEE, Richard. 2007. Food Security and Food Sovereignty. Centre for Rural
Economy Discussion Paper Series No. 11. Universidad de Newcastle bajo Tyne. http://www.ncl.ac.uk/cre/publish/discussionpapers/pdfs/dp11\%20Lee.pdf 